\title{
Cell-free miR-17-5p as a diagnostic biomarker for gastric cancer inhibits dendritic cell maturation
}

This article was published in the following Dove Medical Press journal:

OncoTargets and Therapy

\author{
Zi-Jin Cui ${ }^{1,2}$ \\ Xiao-Li Xie' \\ Wei $\mathrm{Qi}^{\prime}$ \\ Yi-Chao Yang' \\ Yun $\mathrm{Bai}^{2}$ \\ Jing $\operatorname{Han}^{\prime}$ \\ Qian Ding' \\ Hui-Qing Jiang' \\ 'Department of Gastroenterology, \\ Hebei Key Laboratory of \\ Gastroenterology, Hebei Institute \\ of Gastroenterology, The Second \\ Hospital of Hebei Medical \\ University, Shijiazhuang, People's \\ Republic of China; ${ }^{2}$ Department of \\ Gastroenterology, Hebei General \\ Hospital, Shijiazhuang, People's \\ Republic of China
}

Purpose: Gastric cancer (GC) patients display aberrant miRNA expression and defective dendritic cell function. However, the role of cancer cell-derived oncomiR in GC detection and dendritic cell (DC) maturation remains largely elusive.

Methods: Candidate miRNAs were selected by deep sequencing ( $8 \mathrm{GC}$ plasma samples vs 8 control plasma samples; 8 GC tissues vs 8 adjacent normal gastric tissues) and confirmed by PCR with 164 plasma samples and 72 formalin-fixed paraffin-embedded GC tissue samples. Their diagnostic performance was evaluated by receiver operating characteristic curve. Cy3 fluorescence signals in DCs, exposed to conditioned medium obtained from BGC-823 cells pre-transfected with Cy3-miR-17-5p, were determined by flow cytometry and visualized by confocal microscopy. Functional and phenotypical alterations of DCs were assayed when DCs were transfected with miR-17-5p in vitro.

Results: Deep sequencing and RT-PCR confirmed that five shared miRNAs were upregulated in plasma and tissue samples of GC patients. Cell-free miR-17-5p was superior to others in GC detection with an area under the curve of 0.82 , and correlated with lymphatic metastasis and poor overall survival. GC cell-shuttled miR-17-5p can be delivered to immature DCs, and they significantly inhibited LPS-stimulated phenotypic maturation by diminishing the expression of maturation markers (MHC II, CD80 and CD86 molecules). In line with those alterations in the phenotypic markers, functional experiments demonstrated that miR-17-5p triggered an inhibitory effect on DCs endocytic activity and decreased tumor necrosis factor- $\alpha$ and IL-12 secretion, while enhancing IL-10 production. Mixed lymphocyte reaction showed that miR17-5p inhibited the T cell stimulating effect of DCs and favored regulatory T cells expansion. Conclusion: GC cell-derived miR-17-5p is a potential biomarker for GC detection. Taken up by DCs, miR-17-5p weakened antitumor immune responses via inhibiting the maturation of dendritic cells.

Keywords: gastric cancer, cell-free miRNA, biomarker, intracellular communication, dendritic cell

\section{Introduction}

Gastric cancer (GC) is an extremely aggressive malignancy with high incidence and mortality rate. ${ }^{1}$ Limited success was achieved in GC therapy because of a lack of early detection and effective treatment. Researches revealed that cancer cell-derived miRNAs indicate its status and promotes intercellular communication between cancer cells and immune cells living in the tumor microenvironment, which decides tumor outcome. ${ }^{2,3}$

MiRNAs are frequently dysregulated in many malignancies, acting as oncogenes or tumor suppressive genes. As the spectrum between intracellular and extracellular
Correspondence: Hui-Qing Jiang

Department of Gastroenterology, Hebei Key Laboratory of Gastroenterology, Hebei Institute of Gastroenterology, The Second Hospital of Hebei Medical University, 215 Heping West Road, Shijiazhuang, Hebei 050000, People's Republic of China

Tel +863 II6 6002955

Email jianghq@aliyun.com (c)
hereby accept the Terms. Non-commercial uses of the work are permitted without any further permission from Dove Medical Press Limited, provided the work is properly attributed. For permission for commercial use of this work, please see paragraphs 4.2 and 5 of our Terms (https://www.dovepress.com/terms.php). 
miRNA is unparalleled, only a small part of extracellular miRNAs reflects the dynamics of its parental cell, which few studies focused on. Dendritic cells (DCs) initiate or silence $\mathrm{T}$ cell immune responses based on their state of activation and maturation. In tumor context, DCs are transformed into negative regulator of immunity and help tumor evade immunological surveillance. Accumulating evidence shows that the maturation and function of DCs are mediated by miRNAs. ${ }^{4}$ Tumor-derived miRNAs can be taken up by DC, mediating target gene expression and participating in the regulation of tumor immunity. ${ }^{5,6}$

GC patients display aberrant miRNA expression intracellularly and extracellularly, and they are featured with DC dysfunction and regulatory $\mathrm{T}$ cells (Tregs) infiltration. ${ }^{7-9}$ While little is known about the GC-derived oncogenic miRNAs in diagnostic utility and DC function. This study is to screen for the oncogenic miRNA of tumor cell origin, investigate its role in the detection of $\mathrm{GC}$ and its effect on the phenotypic and functional maturation of DC.

\section{Methods}

\section{Clinical sample collection and ethnic consideration}

This study involved 180 plasma samples and 88 formalinfixed paraffin-embedded (FFPE) GC tissues were collected from Clinical Sample Preservation Center of the Second Hospital of Hebei Medical University. Use of these samples and related patient information was approved by patients or their legal representative. Patients' informed consent was obtained. This research was approved by the Ethics Committee of the Second Hospital of Hebei Medical University. The clinical characteristics were summarized in Table S1.

All authors confirm that the patient consent was written informed consent, and that this was conducted in accordance with the Declaration of Helsinki.

\section{GC cell lines and generation of DCs}

Human GC cell lines (BGC-823, SGC-7901) were purchased from Chinese Academy of Sciences (Shanghai, China). All cells were maintained in RPMI-1640 medium (Thermo Fisher Scientific, Waltham, MA, USA) supplemented with 10\% FCS (Biological Industries, 1 Beit-Haemek, Israel) and $1 \%$ penicillin and streptomycin (Thermo Fisher Scientific).

Human monocyte-derived DCs (moDCs) were isolated from buffy coat of healthy blood donors as described previously. ${ }^{10}$ Bone marrow-derived dendritic cells (BMDCs) were isolated from progenitors of C57BL/6 mice. ${ }^{11} \mathrm{CD} 11 \mathrm{c}^{+}$ cells at fifth day were positively isolated by magnetic microbeads (BD Biosciences, San Jose, CA, USA) with purity $>90 \%$ as confirmed by flow cytometry. Experiments were performed with the approval of the Scientific Investigation Board of The Second Hospital of Hebei Medical University (Shijiazhuang, China).

Purified BMDCs were seeded onto 24-well plates, and stimulated with LPS (mature DC), untreated (immature DC [imDC]), or treated with miR-17-5p mimics/scramble control with Lipofectamine RNAIMAX (Thermo Fisher Scientific). Cells were incubated for 12 hours and then lipopolysaccharide (LPS) was added to induce maturation for another 12 hours. The efficiency of transfection was validated by RT-PCR.

\section{RNA isolation and quantification by deep sequencing and RT-PCR}

MiRNA was extracted with miRcute miRNAs Isolation Kit (Tiangen, Beijing, China) or miRNeasy FFPE Kit (Qiagen, Hamburg, Germany). MRNA was isolated by TRIzol-based procedure (Tiangen). Deep sequencing was entrusted to Beijing Genomics Institute, and four small RNA libraries were constructed with the following four mixed samples: 8 GC plasma samples, 8 healthy control plasma samples, 8 GC tissues and 8 adjacent normal gastric tissues.

The relative expression level of indicated miRNAs and mRNAs were assessed by RT-PCR using SYBR-Green-based qPCR assays (Qiagen). The relative quantity was calculated using the $2^{-\Delta \Delta C t}$ method. Cellular and cell-free miRNA expression was normalized to RUN6B and cel-miR-39-3p, respectively. Normalization for mRNA was performed with $\beta$-actin.

\section{FITC-dextran uptake assay}

In order to explore endocytosis of miR-17-5p-treated DC, $1 \mathrm{mg} / \mathrm{mL}$ FITC-dextran (Sigma-Aldrich Co., St Louis, MO, USA) was added to pretreated DCs $\left(5 \times 10^{5}\right)$ followed by incubation for 1 hour at $37^{\circ} \mathrm{C}$ or $4^{\circ} \mathrm{C}$ (background) without light. Fluorescence intensities within cells were analyzed with flow cytometry.

\section{Flow cytometric analysis}

BMDCs purity and phenotype were analyzed with the following anti-mouse Abs: PE-CD11c, APC-CD80, FITCCD86 and PE/CY7-MHC II. MoDCs were stained with APC-CD11c. For Tregs analysis, cells were primarily stained with FITC-CD4, PE-CD25, and then with Alexa 647-Foxp3 after permeabilization with transcription factor buffer set (all from BD Biosciences). Before each staining, anti-CD16/32 was included to block Fc. Stained cells were analyzed with BD FACSVerse. Data were acquired and analyzed with FlowJo software (Ashland, OR, USA). ${ }^{31}$ 


\section{Cytokine assays}

Pretreated DCs and their supernatants were harvested for further analysis. PCR was used to evaluate cytokine productions of DCs. Collected supernatants were centrifuged to remove cell debris before being stored at $-80^{\circ} \mathrm{C}$, and ELISA was performed to explore cytokine expressions (Multi Sciences, Beijing, China). These assays were performed in triplicate.

\section{Mixed lymphocyte reaction (MLR) assay}

$\mathrm{CD}^{+} \mathrm{T}$ cells were isolated from splenocytes of $\mathrm{BALB} / \mathrm{c}$ mice with anti-CD4 magnetic particles (BD Biosciences). On passing through columns (BD Biosciences), the labeled $\mathrm{CD}^{+}{ }^{+} \mathrm{T}$ cells were purified to $\sim 99 \%$. To determine $\mathrm{T}$ cell priming capacities, DCs were pretreated with mitomycin C and added at different densities (10:1, 20:1, 50:1 and 100:1) to $\mathrm{T}$ cells for 3 days. BrdU ELISA was used to determine $\mathrm{T}$ cell proliferation according to the manufacturer's instructions (Merck Millipore, Billerica, MA, USA). Tregs concentration in the coculture system was quantified by flow cytometry as described above.

\section{Statistical analyses}

Student's $t$-test or Wilcoxon signed-rank test was used for comparison between two groups, and Kruskal-Wallis test followed by Bonferroni correction was used for comparison between multiple groups. A $P$-value $<0.05$ was considered to be significant. Correlation assay was performed by Spearman's correlation. The receiver operating characteristic (ROC) curve was used to explore the potential diagnostic utility. Survival analysis was performed by the Kaplan-Meier method and analyzed with log-rank test. Statistical analyses were performed with SPSS 19.0 (IBM Corporation, Armonk, NY, USA) and GraphPad Prism 6 (GraphPad Software, Inc., La Jolla, CA, USA).

\section{Results \\ Study design}

OncomiRs are upregulated in cancer cells and some of them act as noninvasive biomarker when released in peripheral blood and even as intercellular transmitter upon received by target cells. This study is designed to identify cancer cell-derived oncomiRs, and investigate their role in GC detection and DC maturation, and it is illustrated as follows: (1) Screening for upregulated miRNAs in GC patients by deep sequencing. (2) Validation of candidate miRNAs by RT-PCR in 180 plasma samples and 88 tissue samples and assessment of its diagnostic performance using ROC analysis. (3) Functional experiment: the impact of GC cell-derived miRNA on imDCs (Figure S1).

\section{MiRNAs screening and confirmation}

First, we sequenced miRNA in pooled plasma and FFPE samples and obtained four unique miRNA profiles. Candidate miRNAs were further selected if showed more than 2-fold upregulated expression in GC plasma and tissue samples.

As a result, there were 39 shared differentially expressed miRNAs in tissue and plasma samples of GC patients, of which 31 were upregulated (Figure 1). As confirmed by RT-PCR with each sample of the pooled one, five miRNAs (miR-17-5p, miR-127-3p, miR-379-5p, miR-433-3p, miR-654-3p) were consistent with the findings of the deep sequencing.

\section{Validation of the five miRNAs}

To verify the differential expression of the five candidate miRNAs in GC patients, an independent cohort (42 GC plasma samples vs 42 healthy control plasma samples; 36 GC tissues vs 36 adjacent normal gastric tissues) was included. Compared to controls, all studied plasma miRNA showed a higher expression in GC samples, and miR-17-5p was the highest dysregulated miRNA in plasma with up to 6.75 -fold expression difference (Table 1). The differential expressions of the five miRNAs were also found in FFPE samples (Figure 1C). The differential expression patterns of the five candidate miRNAs were concordant between the training phase and the validation phase.

\section{Diagnostic value of plasma miRNA classifiers in GC}

Of the five miRNAs, only miR-17-5p was reported oncogenic in GC development previously. ${ }^{12}$ To evaluate miRNA origin, BGC-823 cells were cultured in serum-free media for 48 hours, and supernatants were collected for miRNA expression analysis. Expression of MiR-17-5p but not others in media containing BGC-823 increased with incubation time, suggesting that miR-17-5p may be derived from GC cells, while others may be from stromal cells or byproducts of cell communication in the microenvironment (Figure S2). The same trend was also observed with SGC-7901.

Further expression validation of the five miRNAs was done in a larger cohort ( $82 \mathrm{GC}$ plasma samples vs 82 healthy control plasma samples). In line with previous findings, miR-17-5p showed the highest expression difference (8.99-fold) compared to controls (Figure 2A). The elevated expression of miR-17-5p began at early stage and enhanced stepwise with higher tumor stage (Figure 2B). We then examined the potential diagnostic utility of the five miRNAs in discriminating $\mathrm{GC}$ patients from cancer-free subjects with ROC analysis (Figure 2C). MiR-17-5p displayed the highest AUC of 0.82 (95\% CI: $0.75-0.88$ ), and the cutoff of 0.015 
A

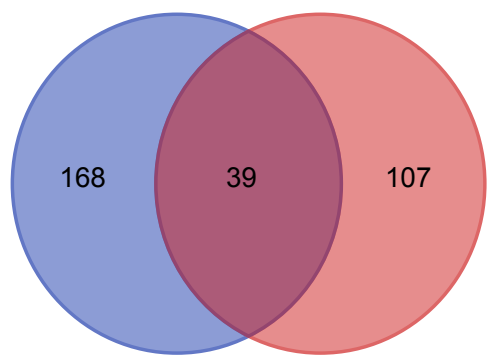

Tissue

Plasma
B

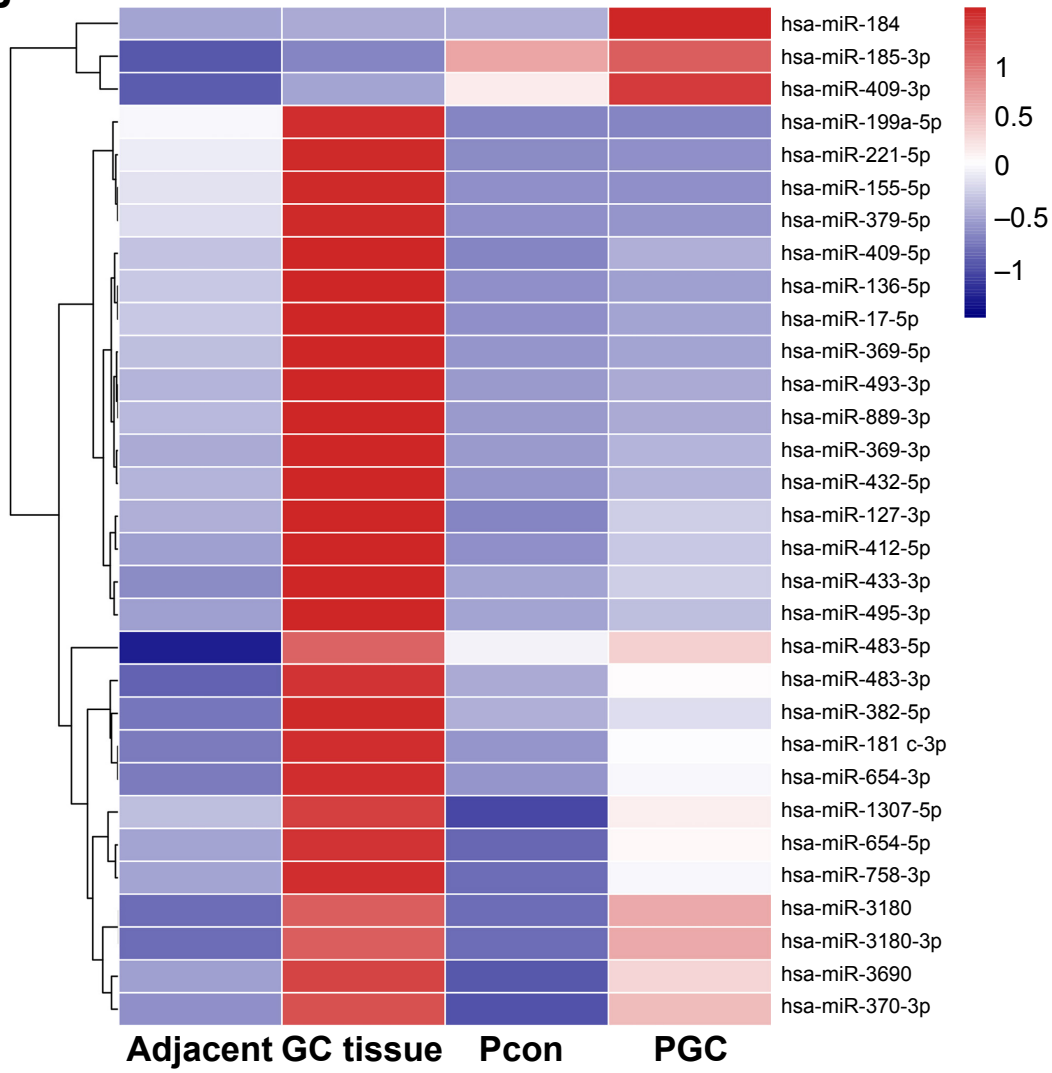

\begin{tabular}{lll} 
Name & Fold change in plasma & Fold change in tissue \\
\hline MiR-17-5p & 6.75 & 4.25 \\
MiR-127-3p & 3.32 & 2.36 \\
MiR-379-5p & 2.75 & 5.97 \\
MiR-433-3p & 6.29 & 3.37 \\
MiR-654-3p & 2.96 & 3.12 \\
\hline
\end{tabular}

Figure I Differentially expressed miRNAs in tissue and plasma samples of GC patients. (A) Venn diagram of the number of special and shared miRNAs with $\geq 2$-fold alteration in tissue and plasma samples revealed by deep sequencing. (B) Hierarchical clustering showed the 3I miRNAs upregulated both in plasma and tissue samples. (C) Expression levels of the 31 miRNAs were examined by PCR in deep sequencing samples and then in 84 plasma and 72 tissue samples. Five upregulated miRNAs (miR-175p, miR-127-3p, miR-379-5p, miR-433-3p, miR-654-3p) were selected for further analysis.

Abbreviations: GC, gastric cancer; Pcon, control plasma; PGC, GC plasma.

would provide the sensitivity of $65.90 \%$ and specificity of 98.8\% (Table S2). Summarily, our results indicated that oncogenic miR-17-5p was shuttled by GC cells, and it was superior to other candidate miRNAs in GC detection.

Table I Relative expression levels of the five miRNAs in plasma samples

\begin{tabular}{|l|l|l|l|}
\hline \multirow{2}{*}{ Name } & \multicolumn{2}{|l|}{ Relative expression level } & \multirow{2}{*}{ P-value } \\
\cline { 2 - 3 } & $\begin{array}{l}\text { Healthy } \\
\text { control }\end{array}$ & $\begin{array}{l}\text { Gastric cancer } \\
\text { patient }\end{array}$ & \\
\hline MiR-17-5p & $0.0074 \pm 0.0084$ & $0.024 I \pm 0.0425$ & 0.00 \\
\hline MiR-127-3p & $0.0139 \pm 0.0113$ & $0.0226 \pm 0.0123$ & 0.00 \\
\hline MiR-379-5p & $0.0186 \pm 0.0120$ & $0.0335 \pm 0.0416$ & 0.00 \\
\hline MiR-433-3p & $0.0266 \pm 0.0406$ & $0.0754 \pm 0.0954$ & 0.00 \\
\hline MiR-654-3p & $0.0033 \pm 0.0049$ & $0.0097 \pm 0.0101$ & 0.00 \\
\hline
\end{tabular}

\section{Correlation between plasma miR-17-5p expression and GC progression}

The progression of GC patients is closely related to pathological stage and metastasis. Though there was an increased trend for miR-17-5p expression with pathological stage, no significant correlation was found between them. Further analysis found that higher miR-17-5p expression was significantly associated with lymph node metastasis but not distant metastasis (Table 2). Kaplan-Meier curve was plotted for survival analysis and the median follow-up time was found to be 33 months (range: $27-53$ months). The 90 GC patients were divided into two groups based on the Youden's index in ROC analysis and results revealed that $\mathrm{GC}$ patients with high miR-17-5p level had a lower overall survival time than those 

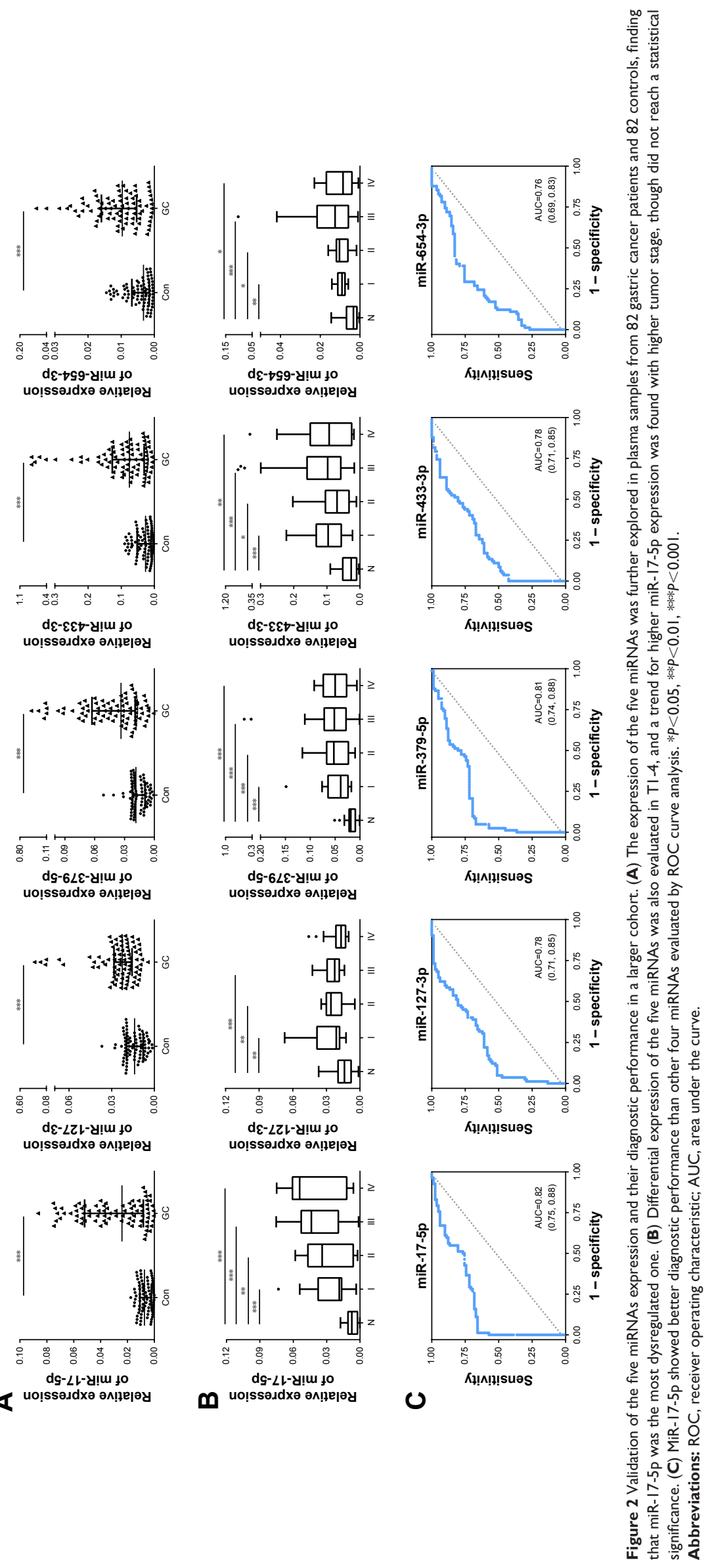
Table 2 Relationship between miR-17-5p expression and clinical variables of gastric cancer patients

\begin{tabular}{|l|l|l|}
\hline & $r_{s}$ & $P$-value \\
\hline Pathological stage & 0.23 & 0.07 \\
\hline Lymph node metastasis & 0.54 & 0.00 \\
\hline Distant metastasis & 0.15 & 0.23 \\
\hline
\end{tabular}

with low miR-17-5p level (Figure 3). Our results suggested that miR-17-5p might play a role in the progression of GC.

\section{GC cell-derived miR-17-5p was taken up by immature DCs}

Emerging evidence suggests that tumor-originated miRNAs play an important role in shaping DCs, phenotype and function. ${ }^{13}$ For GC patients, imDC density was high in peripheral blood and tissues, which correlated with a disappointing clinical outcome. ${ }^{14,15}$ In the natural history of DC maturation, the expression of miR-17 was reduced..$^{16}$ Thus, we hypothesized that GC cell-derived miR-17-5p inhibited DC maturation to promote GC development. To validate the hypothesis, we further confirmed the release of miR-17-5p by GC cells and detected whether the cancer-derived miR17-5p can be taken up by imDCs.

We plated BGC-823 in three different numbers and the medium was changed at 6 hours after plating. Medium was harvested 24 hours and 48 hours thereafter. An increased tendency of miR-17-5p concentration was confirmed with a longer interval when cells were planted at a low density. In addition, a significant positive correlation was confirmed between miR-17-5p concentration and plated cell number

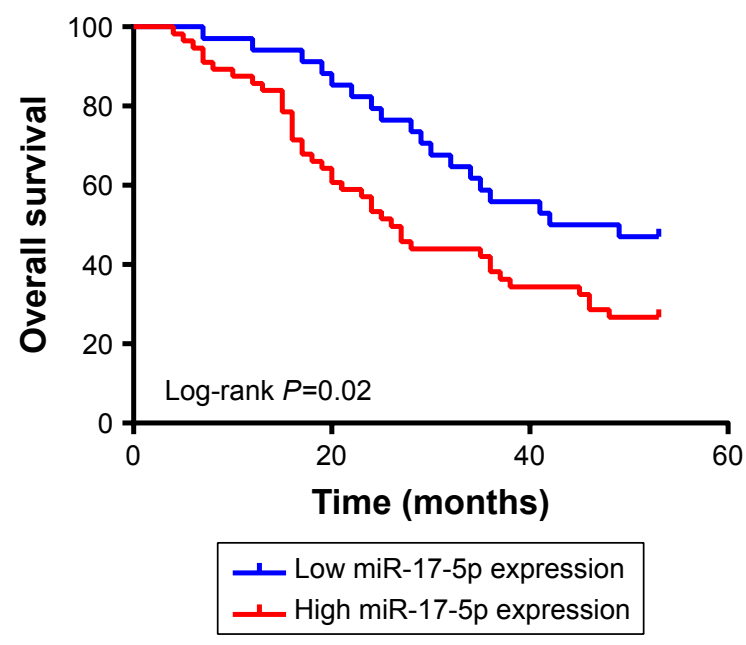

Figure 3 Kaplan-Meier curves for overall survival stratified by miR-17-5p expression. Gastric cancer patients with high miR-17-5p expression showed shorter overall survival than those with low miR-17-5p expression ( $P=0.02$, Log-rank test). at 24 hours $\left(R^{2}=0.96, P=0.00\right)$ (Figure $\left.\mathrm{S} 3 \mathrm{~A}\right)$. We then transfected GC cells with transfection reagent (mock GC), miR-17-5p (miR-17 GC), or Cy3-miR-17-5p (Cy3-miR-17 GC), and collected the conditioned medium to coculture with imDCs for 24 hours. Flow cytometry analysis indicated a percentage of $27.6 \%$ in cy 3 positive imDCs, when co-cultured with conditioned medium with $\mathrm{Cy} 3-\mathrm{miR}-17 \mathrm{GC}$. Only trace cy3 signal was detected in imDCs cocultured with the same form of mock GC (Figure S3B). Furthermore, the internalized Cy3-miR-17-5p can be visualized by fluorescence microscopy analysis (Figure S3C). These data indicated that miR-17-5p within GC cells can be transferred to imDCs.

\section{MiR-17-5p pretreatment depressed LPS- induced DC phenotype maturation}

Since DCs ardently take up GC cell-derived miR-17-5p from their environment, we transfected DC with miR-17-5p to mimic the influence of GC on DC.

ImDCs were pretreated with miR-17-5p mimic, or scramble control for 12 hours before being matured. In unprimed cells, the expression levels of CD80, CD86 and MHC-II were enhanced by LPS stimulation. However, miR-17-5p pretreatment significantly depressed LPS-induced elevation of CD80, CD86 and MHC-II expression (Figure 4A). Therefore, overexpression of miR-17-5p impaired LPS-stimulated DC maturation in phenotype.

\section{MiR-I7-5p attenuated endocytic activity of DCs}

ImDCs show potent capacity of phagocytosis, and this functional characteristic decreases during maturation. Flow cytometry showed that FITC-dextran and $\mathrm{CD} 11 \mathrm{c}$ positive cells were less than $10 \%$ at $4{ }^{\circ} \mathrm{C}$, indicating a specific FITC-dextran uptake of DCs. DCs uptake of FITC-dextran was significantly reduced by LPS, while this reduction was dramatically attenuated by pretreatment with miR-17-5p (Figure 4B). Therefore, the efficacy of miR-17-5p in stalling DCs maturation mirrors their ability in inhibiting phenotypic maturation.

\section{MiR-I7-5p modulated the cytokine profile of DCs upon LPS stimulation}

Cytokines produced by DCs, acting as antitumor or tumorpromoting mediators, determine their functional status. To explore the effect of miR-17-5p on cytokine profile of DC in the process of maturation, the cytokine expression levels in cells and its supernatants were measured using specific RT-PCR and ELISA. Compared to control-dendritic cells (con-DCs), a decrease in tumor necrosis factor- $\alpha$ expression 
A

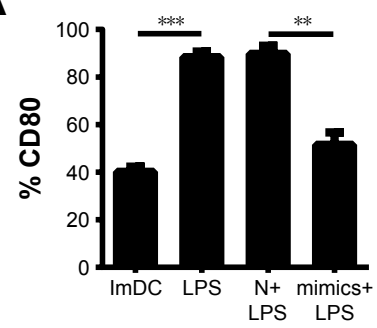

C

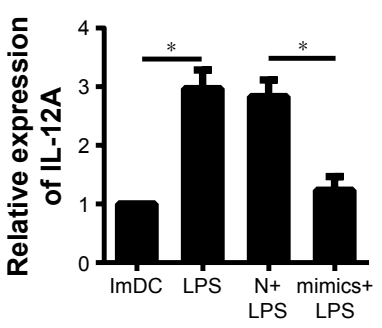

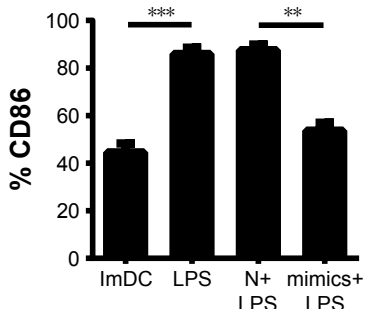

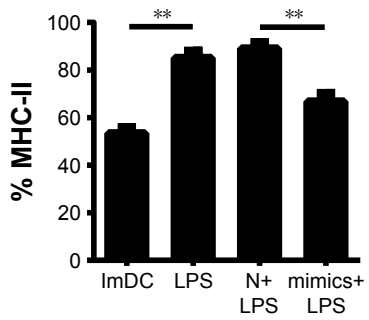

B

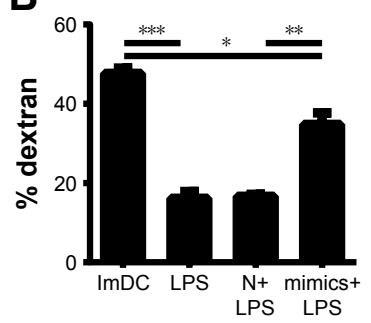

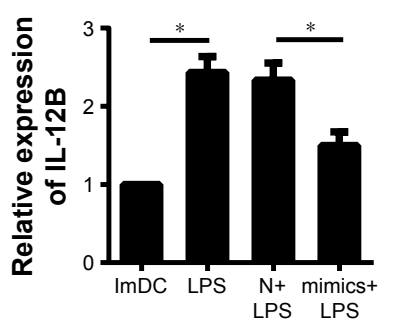
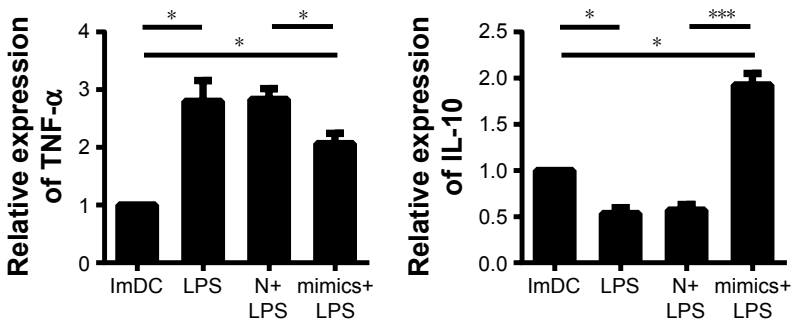

IL-12
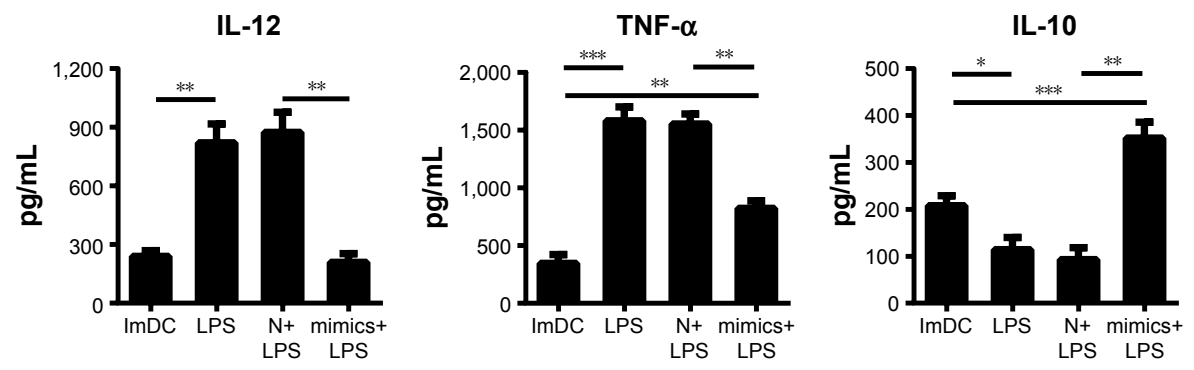

Figure 4 Overexpression of miR-17-5p functionally hampered DC maturation. ImDCs were transfected with miR-17-5p mimics or scramble control and then stimulated with LPS. (A) LPS-induced upregulation of CD80, CD86 and MHC II expression was significantly attenuated by miR-17-5p pretreatment detected by flow cytometry gating on $\mathrm{CDIIc}$. (B) Endocytic activity of DC was enhanced by miR- I7-5p as measured by flow cytometry. The raw $\triangle \mathrm{MFI}$ value $\left(37^{\circ} \mathrm{C} \mathrm{MFI} \mathrm{to} 4{ }^{\circ} \mathrm{C} \mathrm{MFI)} \mathrm{for} \mathrm{each} \mathrm{condition} \mathrm{is} \mathrm{shown.}\right.$ (C) MiR-17-5p induced lower level of IL- 12 and TNF- $\alpha$ but higher level of IL- 10 in DCs than control. $* P<0.05, * * P<0.01$, $* * * P<0.001$.

Abbreviations: MFI, mean fluorescence intensity; N, scramble; imDC, Immature dendritic cell; LPS, lipopolysaccharide; TNF- $\alpha$, tumor necrosis factor- $\alpha$.

level was detected in miR-17-DCs, which could further downregulate the expression of co-stimulators. Next, we investigated the expression of the antitumor cytokine IL-12 and the tumor-promoting cytokine IL-10. Compared with con-DC, miR-17-DCs produced a higher level of IL-10 and a lower level of IL-12 (Figure 4C). The results above suggested that miR-17-5p may affect tumor immunity by mediating cytokines production of DCs.

\section{MiR-17-5p-pretreated DC induced T cell hyporesponsiveness and Tregs expansion in MLRs}

To elucidate the effect of miR-17-DCs on triggering $\mathrm{T}$ cell response, pretreated DCs were cocultured with $\mathrm{T}$ cells for 3 days. DCs stimulated with LPS triggered strong $\mathrm{T}$ lymphocyte proliferation, while this response was suppressed by miR-17-5p pretreatment of DC (Figure 5A). In addition, we observed that the $\mathrm{CD} 4^{+} \mathrm{CD} 25^{+}$Foxp $3^{+} \mathrm{T}$ cells were decreased by LPS stimulation but was increased by
miR-17-DCs. MiR-17-DC-stimulated T cells even displayed higher Tregs percentage than imDCs when at 1:10 (DC: T cell) (Figure 5B). Thus, DCs transfected with miR-17-5p mimics were potent inducers of Tregs.

These data indicated that overexpression of miR-17-5p blocked DC maturation in phenotype and function, and made it difficult to induce $\mathrm{T}$ cell proliferation and instead supported Tregs expansion.

\section{Discussion}

In our present study, we investigated the role of tumorderived oncomiR in the detection of GC and cell-cell communication. Deep sequencing revealed that miR-17-5p was significantly elevated in GC tissue and plasma samples and it further confirmed a tumor cell origin by cell-based assay. MiR-17-5p showed potent advantage in differentiating tumor lesions from the normal ones. In vitro experiment showed that miR-17-5p can be taken up by DC, and its inhibitory effect was found on the LPS-induced maturation of DC, 


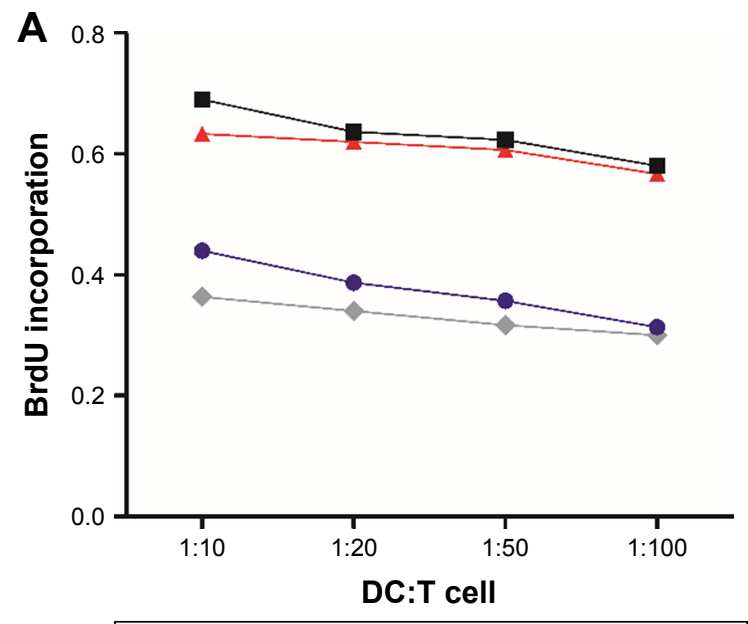

- ImDC $\because$ LPS $\rightarrow \mathrm{N}+\mathrm{LPS} \multimap$ mimics+LPS

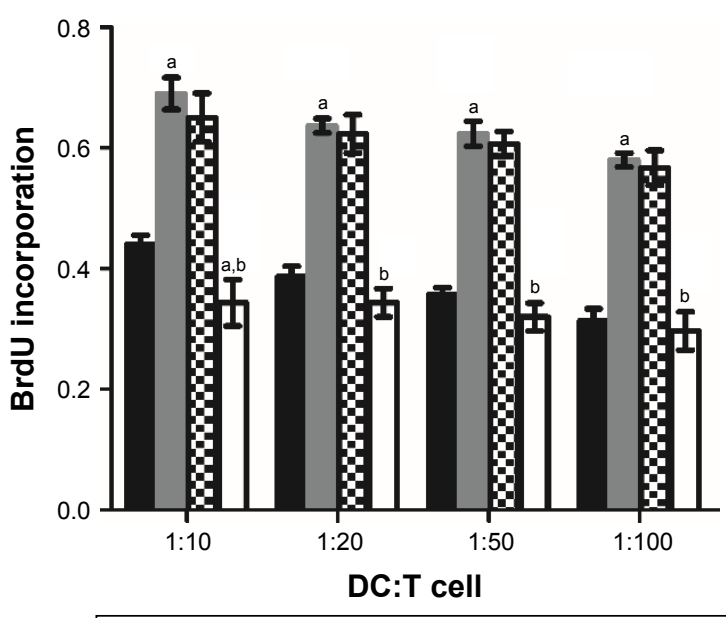

ImDC LPS
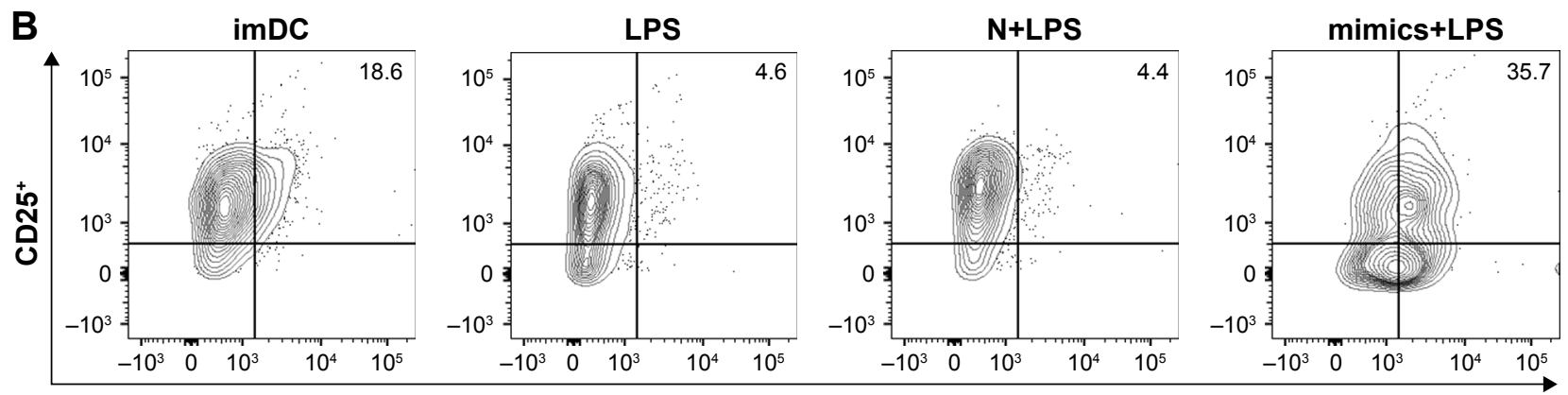

Foxp $^{+}$

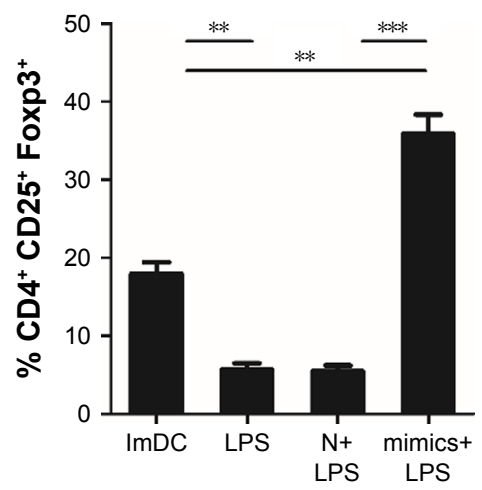

Figure 5 MiR-I7-DC reduces T cell proliferation and favored Treg expansion. Pretreated BMDCs were added at different densities (I0:I, 20:I, 50:I and I00:I) to T cells for 3 days and incubated with BrdU (24 hours) to quantify proliferation. (A) LPS-stimulated DCs triggered strong T lymphocyte proliferation, whereas this response was suppressed by miR-17-5p pretreatment. (B) Tregs $\left(C D 4^{+} C D 25^{+}\right.$Foxp $\left.3^{+}\right)$were decreased by LPS stimulation but was increased by miR-I7-DCs. At high concentration, miR-17-DC-stimulated T cells displayed higher Tregs percentage than imDCs. a Compared to imDCs. ${ }^{b}$ Compared to $N+L P S$. $* * P<0.0$ I, $* * * P<0.001$.

Abbreviations: BMDC, bone marrow-derived dendritic cell; N, scramble; imDC, immature dendritic cell; LPS, lipopolysaccharide; Treg, regulatory T cells.

by exploring the expression of surface markers, cytokine production, endocytic activity, and communication between the DC and T cells.

MiRNAs are small regulatory RNAs that play a critical role in GC progression and tumor immunity. Cellular oncomiRs are overexpressed in tumor cells, and only a small part of extracellular miRNAs is paralleled with their origin cells.
Five miRNAs (miR-17-5p, miR-127-3p, miR-379-5p, miR433-3p and miR-654-3p) were confirmed to be upregulated in GC plasma and tissue samples, among which miR-17-5p was the most studied. As a member of miR-17-92 cluster, miR-17-5p enhances tumor properties and induces chemoresistance. ${ }^{12,17}$ We further explored the potential origin of the five candidate miRNAs, and found that only miR-17-5p was 
secreted by GC cells. MiR-17-5p also performed better than the other four miRNAs in GC detection. A previous study revealed that miR-17-5p expression was correlated with GC differentiation and was reduced after operation. ${ }^{18}$ In line with previous findings, we found that miR-17-5p also was correlated with lymphatic metastasis and poor overall survival, which all triggered our investigation of its novel role in tumor immunity.

MiRNAs equipped with exosomes, high-density lipoprotein particles or $\mathrm{AGO} 2$ are capable of inducing functional effects in recipient cells. ${ }^{19,20}$ In 2017, a study found that prostate cancer cell-derived miR-410-5p can be taken up by $\mathrm{DC}$, promoting angiogenesis and tumor growth by decreasing miR-410-3p expression in DC. ${ }^{6}$ While little is known about the effect of GC cell-derived miRNA on DC. Considering the heterogeneity of the extracellular miRNA population, we transfected imDC with miR-17-5p to mimic the influence of tumor-derived miR-17-5p on DC. Overexpression of miR-17-5p inhibited the expression of cell surface molecules in DCs while it enhanced DCs endocytosis, suggesting that miR-17-5p inhibited the transformation of imDCs from antigen-capture cells to antigen-presenting cells. Tumorderived IL-10 showed inhibitory effect on DC maturation, ${ }^{21}$ so the miR-17-5p-induced high IL-10 expression further boosted the immaturity of DCs. IL-10 and IL-12 were inversely produced by DCs. ${ }^{22} \mathrm{IL}-12 \mathrm{p} 70$ is the most important cytokine for Th1 response induction. ${ }^{23}$ IL-10 blocks IL-12p70 production and impairs the ability of DCs to generate Th1 cells. T cells are an important effector of immune system. We observed that miR-17-5p weakened T cell stimulating effect of DCs, owing to reduced expression of surface molecules of DCs and also elevated expression of IL-10, decreased expression of IL-12p70. In GC, an expansion of CD4 ${ }^{+} \mathrm{FOXP}^{+}$ $\mathrm{CD} 25^{\text {high }} \mathrm{T}$ cells was found in $\mathrm{GC}$ mucosa and peripheral blood, which correlated with TNM stage. ${ }^{24,25}$ As to the direct influence of miR-17 on Tregs, controversial conclusion was obtained. ${ }^{26,27}$ Our study showed that miR-17-5p enhanced T cell differentiation to Tregs by downregulating the expression of surface signals on DCs and increasing IL-10 production.

Currently, multiple miRNAs have been investigated as candidate GC biomarker. Studies found that miR-17-5p was upregulated in tissue samples of GC patients, while no consensus has been made about its expression in the peripheral blood. ${ }^{28-30}$ We found that the upregulated miR-17-5p in plasma was superior to other miRNAs in GC detection. The possible reason is that cancer cell-derived molecules more accurately reflect the cancer dynamics. The role of cancer cellderived oncomiR in DC maturation remains largely unknown, and our results indicated that GC cell-derived miR-17-5p can be transferred to DCs, restrained their maturation and finally promoted Treg expansion, which may help tumor escape.

There are several limitations in this study. First, the expression of cell-free miR-17-5p should be further investigated in larger cohorts with different populations using identical extraction and examining method before used clinically, as it can be influenced by various factors, which including genetic background, sample type and test method. Second, the miR-17-5p expression should be further explored in precancerous lesions because early detection and prevention is the key point to reduce mortality rate of GC patients. Third, in vitro functional experiment showed that miR-17-5p inhibited LPS-induced DC maturation, while the underlying mechanism requires further study.

\section{Conclusion}

In summary, we confirm the aberrant expression of plasma and tissue miRNA in GC patients as described previously. In addition we show that cell-free miR-17-5p, originated from GC cells, have better diagnostic performance than other dysregulated miRNAs in GC surveillance. Taken up by imDCs, miR-17-5p inhibits the LPS-induced maturation phenotypically and functionally. Taken together, our data provide vital basis for further investigations on noninvasive GC monitoring and tumor escape, besides, miR-17-5p may be a potential target for gene therapy, which could possibly improve the clinical outcome of GC patients.

\section{Acknowledgments}

This work was supported by Beijing-Tianjin-Hebei Basic Research Cooperation Project (grant number 2018206450), the National Natural Science Foundation of China (grant numbers 81602529 and 81702324), and the National Natural Science Foundation of Hebei Province (grant numbers H2018206176 and H2017206141).

\section{Disclosure}

The authors report no conflicts of interest in this work.

\section{References}

1. Siegel RL, Miller KD, Jemal A. Cancer statistics, 2017. CA Cancer J Clin. 2017;67:7-30. doi:10.3322/caac. 21387

2. Bayraktar R, Van Roosbroeck K, Calin GA. Cell-to-cell communication: microRNAs as hormones. Mol Oncol. 2017;11:1673-1686. doi:10. 1002/1878-0261.12144

3. Fanini F, Fabbri M. Cancer-derived exosomic microRNAs shape the immune system within the tumor microenvironment: state of the art Semin Cell Dev Biol. 2017;67:23-28. doi:10.1016/j.semcdb.2016.12.004

4. Smyth LA, Boardman DA, Tung SL, et al. MicroRNAs affect dendritic cell function and phenotype. Immunology. 2015;144:197-205. doi:10. 1111/imm. 12390 
5. Pyfferoen L, Brabants E, Everaert C, et al. The transcriptome of lung tumor-infiltrating dendritic cells reveals a tumor-supporting phenotype and a microRNA signature with negative impact on clinical outcome. Oncoimmunology. 2017;6:e1253655. doi:10.1080/2162402X.2016.1253655

6. Wang J, Ye H, Zhang D, et al. Cancer-derived circulating MicroRNAs promote tumor angiogenesis by entering dendritic cells to degrade highly complementary MicroRNAs. Theranostics. 2017;7:1407-1421. doi: $10.7150 /$ thno. 18262

7. Hoursu M, Li K, Maskey N, et al. Decreased intratumoral Foxp3 Tregs and increased dendritic cell density by neoadjuvant chemotherapy associated with favorable prognosis in advanced gastric cancer. Int $J$ Clin Exp Pathol. 2014;7:4685-4694.

8. Hoursuang XM, Liu XS, Lin XK, et al. Role of plasmacytoid dendritic cells and inducible costimulator-positive regulatory $\mathrm{T}$ cells in the immunosuppression microenvironment of gastric cancer. Cancer Sci. 2014;105:150-158. doi:10.1111/cas.12327

9. Tsujitani S, Kakeji Y, Watanabe A, et al. Infiltration of dendritic cells in relation to tumor invasion and lymph node metastasis in human gastric cancer. Cancer. 1990;66:2012-2016.

10. Nowatzky J, Manches O, Khan SA, et al. Modulation of human Th17 cell responses through complement receptor 3 (CD11b/CD18) ligation on monocyte-derived dendritic cells. J Autoimmun. 2018;92:57-66. doi:10.1016/j.jaut.2018.05.005

11. Schutt CR, Gendelmanhours E, Mosley RL. Tolerogenic bone marrowderived dendritic cells induce neuroprotective regulatory T cells in a model of Parkinson's disease. Mol Neurodegener. 2018;13:26. doi:10.1186/ s13024-018-0255-7

12. Chen $\mathrm{P}$, Zhao H, Huang J, et al. MicroRNA-17-5p promotes gastric cancer proliferation, migration and invasion by directly targeting early growth response 2. Am J Cancer Res. 2016;6:2010-2020.

13. Zheng J, Jiang HY, Li J, et al. MicroRNA-23b promotes tolerogenic properties of dendritic cells in vitro through inhibiting Notch1/NFkappaB signalling pathways. Allergy. 2012;67:362-370. doi:10.1111/ j.1398-9995.2011.02776.x

14. Lissoni P, Brivio F, Ferrante R, et al. Circulating immature and mature dendritic cells in relation to lymphocyte subsets in patients with gastrointestinal tract cancer. Int J Biol Markers. 2000;15:22-25.

15. Tsukayama S, Omura K, Yoshida K, et al. Prognostic value of CD83positive mature dendritic cells and their relation to vascular endothelial growth factor in advanced human gastric cancer. Oncol Rep. 2005;14: 369-375.

16. Lu C, Huang X, Zhang X, et al. miR-221 and miR-155 regulate human dendritic cell development, apoptosis, and IL-12 production through targeting of p27kip1, KPC1, and SOCS-1. Blood. 2011;117:4293-4303. doi:10.1182/blood-2010-12-322503

17. Wu DM, Hong XW, Wang LL, et al. MicroRNA-17 inhibition overcomes chemoresistance and suppresses epithelial-mesenchymal transition through a DEDD-dependent mechanism in gastric cancer. Int J Biochem Cell Biol. 2018;102:59-70. doi:10.1016/j.biocel.2018. 06.007
18. Wang M, Gu H, Wang S, et al. Circulating miR-17-5p and miR-20a: molecular markers for gastric cancer. Mol Med Rep. 2012;5:1514-1520. doi:10.3892/mmr.2012.828

19. Taghikhani A, Hassan ZM, Ebrahimi M, et al. microRNA modified tumor-derived exosomes as novel tools for maturation of dendritic cells. J Cell Physiol. 2018. doi:10.1002/jcp.27626

20. Zhu JJ, Liu YF, Zhang YP, et al. VAMP3 and SNAP23 mediate the disturbed flow-induced endothelial microRNA secretion and smooth muscle hyperplasia. Proc Natl Acad Sci U S A. 2017;114:8271-8276. doi:10.1073/pnas.1700561114

21. Rizzuti D, Ang M, Sokollik C, et al. Helicobacter pylori inhibits dendritic cell maturation via interleukin-10-mediated activation of the signal transducer and activator of transcription 3 pathway. J Innate Immun. 2015;7:199-211. doi:10.1159/000368232

22. Sondergaard JN, van Heeringen SJ, Looman MWG, et al. Dendritic cells actively limit interleukin-10 production under inflammatory conditions via DC-SCRIPT and dual-specificity phosphatase 4. Front Immunol. 2018;9:1420. doi:10.3389/fimmu.2018.01420

23. Eisenring M, Vom Berg J, Kristiansen G, et al. IL-12 initiates tumor rejection via lymphoid tissue-inducer cells bearing the natural cytotoxicity receptor NKp46. Nat Immunol. 2010;11:1030-1038. doi:10.1038/ni.1947

24. Kindlund B, Sjoling A, Yakkala C, et al. CD4(+) regulatory T cells in gastric cancer mucosa are proliferating and express high levels of IL-10 but little TGF-beta. Gastric Cancer. 2017;20:116-125. doi:10.1007/ s10120-015-0591-z

25. Chen SL, Cai SR, Zhang XH, et al. Expression of CD4+ CD25+ regulatory $\mathrm{T}$ cells and Foxp3 in peripheral blood of patients with gastric carcinoma. J Biol Regul Homeost Agents. 2016;30:197-204.

26. de Kouchkovsky D, Esensten JH, Rosenthal WL, et al. microRNA-17-92 regulates IL-10 production by regulatory $\mathrm{T}$ cells and control of experimental autoimmune encephalomyelitis. J Immunol. 2013;191: 1594-1605. doi:10.4049/jimmunol.1203567

27. Yang HY, Barbi J, Wu CY, et al. MicroRNA-17 modulates regulatory $\mathrm{T}$ cell function by targeting co-regulators of the Foxp3 transcription factor. Immunity. 2016;45:83-93. doi:10.1016/j.immuni.2016.06.022

28. Hoursu G, Lv Q, Yan J, et al. MicroRNA-17 as a promising diagnostic biomarker of gastric cancer: an investigation combining TCGA, GEO, meta-analysis, and bioinformatics. FEBS Open Bio. 2018;8:1508-1523. doi:10.1002/2211-5463.12496

29. Tsujiura M, Ichikawa D, Komatsu S, et al. Circulating microRNAs in plasma of patients with gastric cancers. Br J Cancer. 2010;102: 1174-1179. doi:10.1038/sj.bjc.6605608

30. Zeng Q, Jin C, Chen W, et al. Downregulation of serum miR-17 and miR-106b levels in gastric cancer and benign gastric diseases. Chin J Cancer Res. 2014;26:711-716. doi:10.3978/j.issn.1000-9604. 2014.12.03

31. Zhang S, Ma J, Sheng L, et al. Total coumarins from hydrangea paniculata show renal protective effects in lipopolysaccharide-induced acute kidney injury via anti-inflammatory and antioxidant activities. Frontiers in Pharmacology. 2017;8(872). 


\section{Supplementary materials}

Table SI Characteristics of gastric cancer patients and healthy volunteers

\begin{tabular}{|c|c|c|c|}
\hline \multirow[t]{2}{*}{ Characteristics } & \multirow[t]{2}{*}{ Tissue } & \multicolumn{2}{|l|}{ Plasma } \\
\hline & & Gastric cancer & Healthy control \\
\hline Age (year) & $61.48 \pm 10.49$ & $63.3 \pm 5.33$ & $62.4 \pm 7.24$ \\
\hline \multicolumn{4}{|l|}{ Gender (cases) } \\
\hline Male & 26 & 64 & 64 \\
\hline Female & 14 & 26 & 26 \\
\hline \multicolumn{4}{|l|}{ TNM stage } \\
\hline I & 7 & 12 & - \\
\hline II & 14 & 13 & - \\
\hline III & 11 & 19 & - \\
\hline IV & 8 & 17 & - \\
\hline Uncertain & 0 & 21 & - \\
\hline \multicolumn{4}{|l|}{ Tumor location } \\
\hline Cardia & 7 & 24 & - \\
\hline Fundus & 9 & 12 & - \\
\hline Body & 9 & 17 & - \\
\hline Antrum & 10 & 18 & - \\
\hline Diffuse & 5 & 11 & - \\
\hline \multicolumn{4}{|l|}{ Histology type } \\
\hline Adenocarcinoma & 30 & 64 & - \\
\hline Mucinous adenocarcinoma & 3 & 7 & - \\
\hline Signet-ring cell carcinoma & 4 & 7 & - \\
\hline Neuroendocrine carcinoma & 3 & 4 & - \\
\hline
\end{tabular}




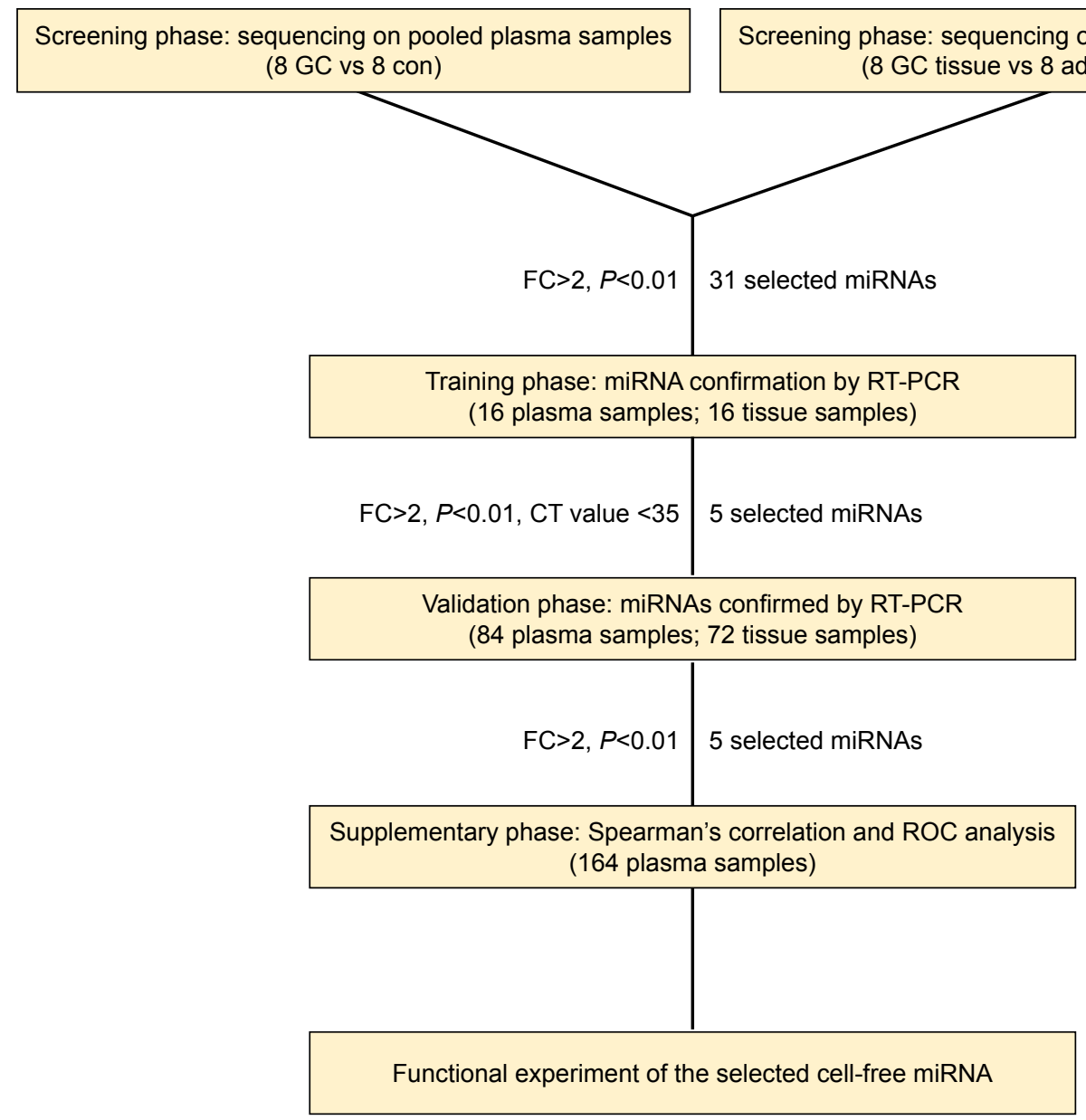

Figure SI Overview of the experiment design.

Abbreviations: CT, cycle threshold; FC, fold change; GC, gastric cancer; ROC, receiver operating characteristic.

A

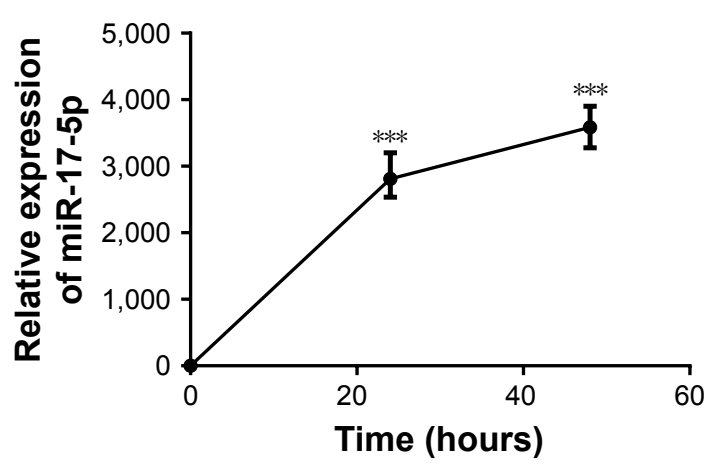

B

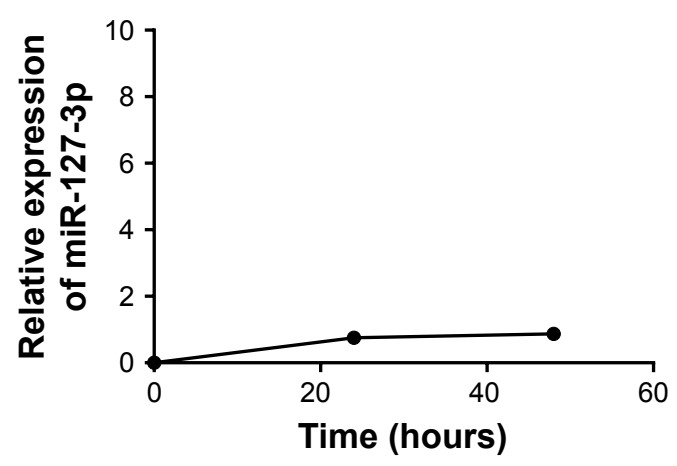

Figure S2 (Continued) 

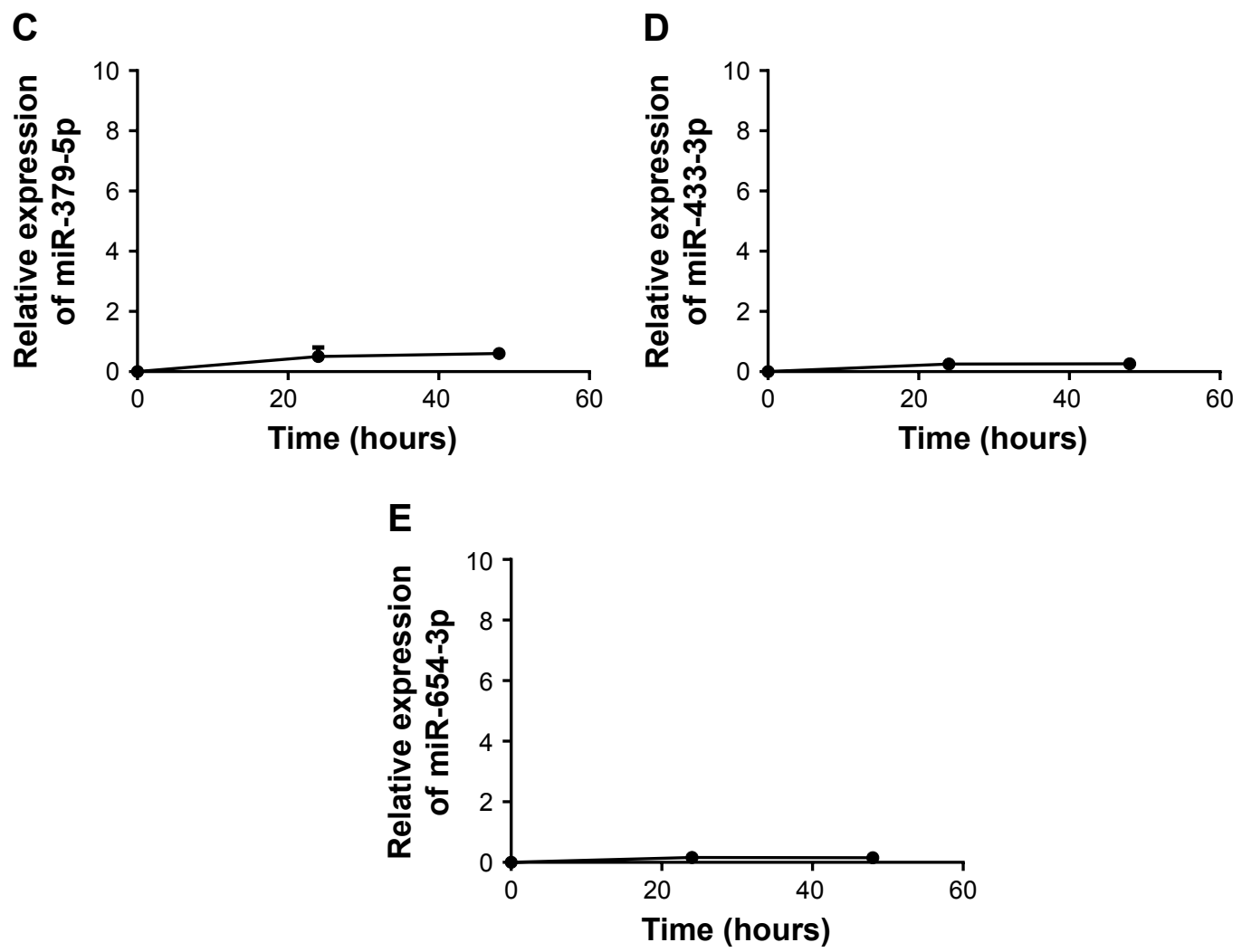

Figure S2 Relative expression levels of identified miRNAs in culture medium of BGC-823 GC cell lines. Expression of miR-17-5p (A) increased with longer incubation time, while miR-I27-3p (B), miR-379-5p (C), miR-433-3p (D) and miR-654-3p (E) levels showed little change. $* * * p<0.00$ I.

Table S2 ROC analysis of the five miRNAs in plasma samples

\begin{tabular}{l|l|l|l|l|l}
\hline Name & AUC & $95 \%$ CI & Cut-off value & Sensitivity & Specificity \\
\hline MiR-17-5p & 0.82 & $0.75-0.88$ & 0.0150 & $65.90 \%$ & $98.80 \%$ \\
MiR-127-3p & 0.78 & $0.71-0.85$ & 0.0223 & $51.20 \%$ & $95.10 \%$ \\
MiR-379-5p & 0.81 & $0.74-0.88$ & 0.0243 & $67.10 \%$ & $95.10 \%$ \\
MiR-433-3p & 0.78 & $0.71-0.85$ & 0.0578 & $61.00 \%$ & $82.90 \%$ \\
MiR-654-3p & 0.76 & $0.69-0.83$ & 0.0056 & $75.60 \%$ & $70.70 \%$ \\
\hline
\end{tabular}

Abbreviations: AUC, area under the curve; ROC, receiver operating characteristic. 

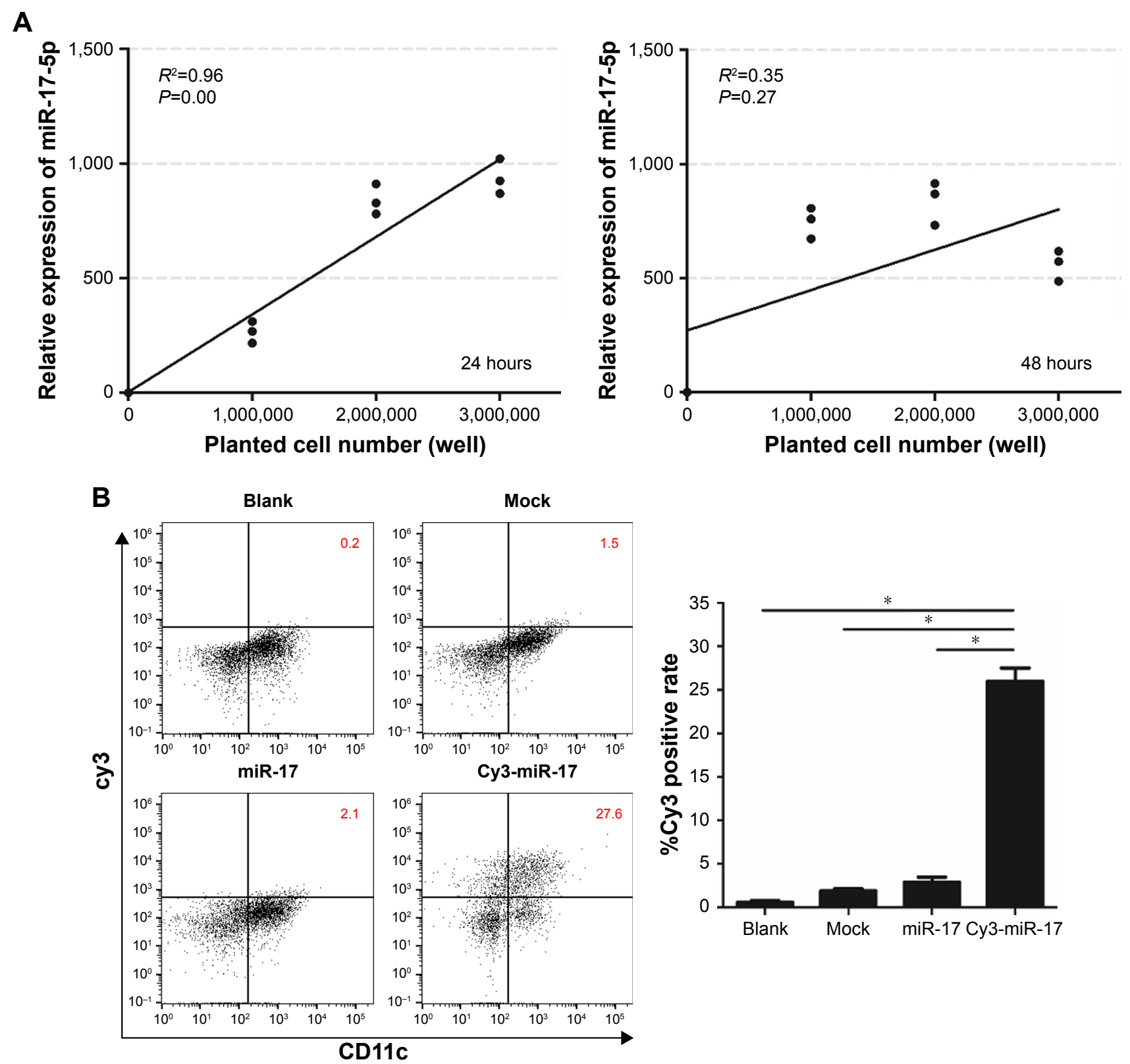

Figure S3 (Continued) 

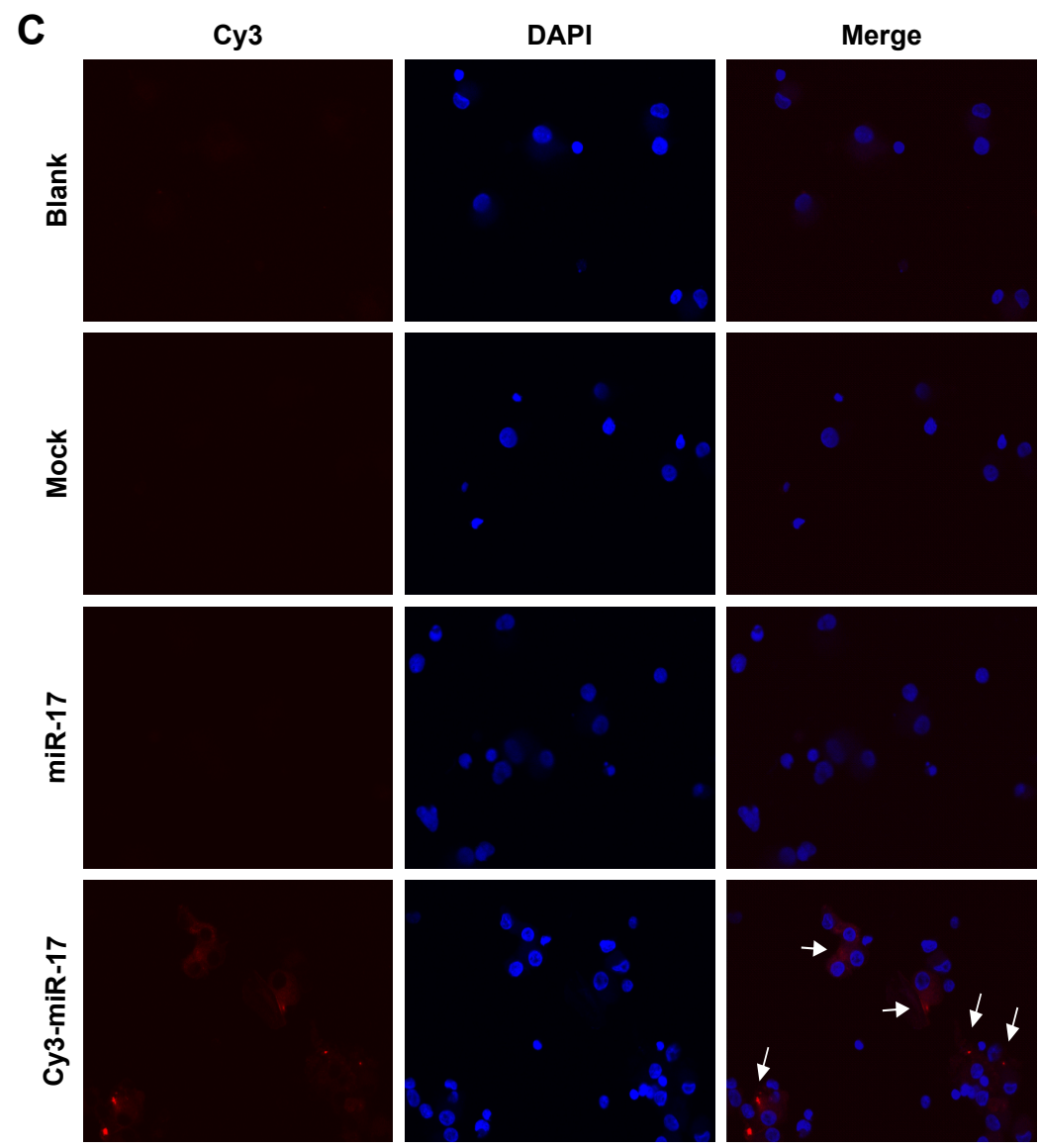

Figure S3 MiR-17-5p is shuttled from gastric cancer cells to imDCs. (A) Medium miR-17-5p concentration was positively related to plated cell number at 24 hours evaluated by Spearman's correlation test. (B) Conditioned medium containing pretreated gastric cancer cells was used to coculture with imDCs for 24 hours, and cy3 positive cells (\%) were quantified by flow cytometry. (C) The cy3 fluorescence signal in imDCs was determined by confocal microscopy. Arrows indicate cy3 signals. Blue fluorescence indicates cell nuclei stained with DAPI.

Notes: Results are presented as means \pm SE from at least three independent tests, $* P<0.00$ I vs indicated group. Blank: untreated; mock: preloaded with transfection reagent; miR-17: preloaded with unlabeled miR-17-5p; cy3-miR-17: preloaded with cy3 fluorescently labeled miR-17-5p.

Abbreviation: imDC, immature dendritic cell.

\section{Publish your work in this journal}

OncoTargets and Therapy is an international, peer-reviewed, open access journal focusing on the pathological basis of all cancers, potential targets for therapy and treatment protocols employed to improve the management of cancer patients. The journal also focuses on the impact of management programs and new therapeutic agents and protocols on

\section{Dovepress}

patient perspectives such as quality of life, adherence and satisfaction. The manuscript management system is completely online and includes a very quick and fair peer-review system, which is all easy to use. Visit http://www.dovepress.com/testimonials.php to read real quotes from published authors. 